\title{
Fibrin Hydrogels Functionalized with Particulated Cartilage Extracellular Matrix and Incorporating Freshly Isolated Stromal Cells as an Injectable for Cartilage Regeneration
}

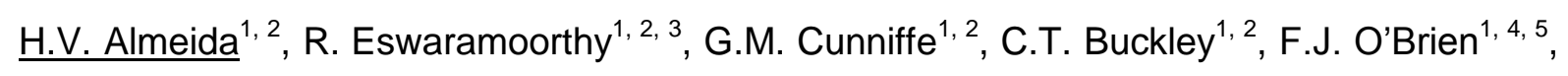
D.J. Kelly ${ }^{1,2,4,5}$

1 Trinity Centre for Bioengineering, Trinity Biomedical Sciences Institute, Trinity College Dublin, Dublin 2, Ireland

${ }^{2}$ Department of Mechanical and Manufacturing Engineering, School of Engineering, Trinity College Dublin, Dublin 2, Ireland

${ }^{3}$ Department of Biomedical Sciences, Sri Ramachandra University, Porur, Chennai, India

${ }^{4}$ Advanced Materials and Bioengineering Research Centre (AMBER), Trinity College Dublin \& Royal College of Surgeons in Ireland, Dublin 2, Ireland

${ }^{5}$ Tissue Engineering Research Group, Department of Anatomy, Royal College of Surgeons in Ireland, Dublin 2, Ireland

${ }^{*}$ Corresponding author: Daniel J. Kelly, Ph.D.

Address: Department of Mechanical and Manufacturing Engineering, School of Engineering, Parson's Building Trinity College Dublin, Dublin 2, Ireland Telephone: +353-1-896-3947, Fax: +353-1-679-5554

E-mail address: kellyd9@tcd.ie 


\section{Abstract}

Freshly isolated stromal cells can potentially be used as an alternative to in vitro expanded cells in regenerative medicine. Their use requires the development of bioactive hydrogels or scaffolds which provide an environment to enhance their proliferation and tissue-specific differentiation in vivo. The goal of the current study was to develop an injectable fibrin hydrogel functionalized with cartilage ECM microparticles and transforming growth factor (TGF)- $\beta 3$ as a putative therapeutic for articular cartilage regeneration. ECM microparticles were produced by cryomilling and freeze-drying porcine articular cartilage. Up to $2 \%(\mathrm{w} / \mathrm{v}) \mathrm{ECM}$ could be incorporated into fibrin without detrimentally affecting its capacity to form stable hydrogels. Even in the presence of such levels of ECM, chondrogenesis of infrapatellar fat pad (IFP)-derived stem cells within these fibrin constructs was enhanced when additionally stimulated with exogenous TGF- $\beta 3$. To further access the chondroinductivity of cartilage ECM, we then compared chondrogenesis of IFP-derived stem cells in fibrin hydrogels functionalized with either particulated ECM or control gelatin microspheres. Cartilage ECM particles could be used to control the delivery of TGF- $\beta 3$ to IFP-derived stem cells within fibrin hydrogels in vitro, and furthermore, led to higher levels of sulphated glycosaminoglycan (sGAG) and collagen accumulation compared to control constructs loaded with gelatin microspheres. In vivo, freshly isolated stromal cells generated a more cartilage-like tissue within fibrin hydrogels functionalized with cartilage ECM particles compared to the control gelatin loaded constructs. These tissues stained strongly for type II collagen and contained higher levels of sGAGs. These results support the use of fibrin hydrogels functionalized with cartilage ECM components in single-stage, cell-based therapies for joint regeneration.

Keywords: Articular Cartilage, Single-Stage Therapy, Fibrin Hydrogel, Extracellular Matrix, Growth Factor. 


\section{Introduction}

Articular cartilage is an avascular tissue with a complex structure that has a limited capacity for self repair [1]. Regenerating articular cartilage still is a challenge in the field of tissue engineering $[1,2]$. Chondral and osteochondral lesions often result in pain and swelling, followed by further joint degeneration and osteoarthritis [1, 2]. Injuries to cartilage can be treated with a range of approaches, including marrow stimulating techniques, mosaicplasty and cell-based therapies [1]. Autologous chondrocyte implantation (ACI) and matrix-induced $\mathrm{ACl}(\mathrm{MACl})$ are examples of currently used techniques in articular cartilage regeneration, which proved to be reasonably successful clinically $[1,3]$. However, these procedures require two surgeries and are significantly more expensive than traditional approaches to articular cartilage repair [4].

A number of different single-stage, cell-based procedures have been proposed that would theoretically overcome the need for two surgical procedures and autologous cell expansion [4-6]. Freshly isolated cells can potentially be obtained from harvested tissue in the surgical room, and efficiently implanted in one single procedure $[5,7,8]$. Freshly isolated stromal cells from the infrapatellar fat pad (IFP), which we have previously shown to be a viable source of chondro-progenitor cells [9-12], are a particularly promising cell type for single-stage procedures. In addition to identifying a suitable cell type, the successful realization of such in-theatre procedures also requires the development of a bioactive scaffold or hydrogel able to promote the proliferation and chondrogenic differentiation of a limited number of multipotent cells, which can be obtained from a donor in one surgical intervention.

Fibrin is a commonly used biomaterial for tissue engineering and is in widespread clinical use, typically as a hemostatic and/or a sealant agent [13]. It has also been investigated as a cell vehicle and as a therapeutic drug delivery system for different tissue engineering applications [13-15]. In the context of articular cartilage tissue engineering, there is evidence to suggest that fibrin is not as chondro-permissive as other well established 
hydrogels [16], with bone marrow and adipose-derived stem cells showing a diminished chondrogenic potential when encapsulated in fibrin [15-17]. There is therefore a clear need for further functionalization of this versatile injectable hydrogel system to optimize its utility for cartilage repair therapies.

Articular cartilage extracellular matrix (ECM)-derived materials have been previously used to engineer cartilage grafts with promising results [11, 12, 18, 19]. In addition, such ECM-derived biomaterials have been used to bind and release chondrogenic factors such as transforming growth factor (TGF)- $\beta 3[11,12]$. Furthermore, ECM particles have also been used to functionalize other biomaterials in an attempt to enhance chondrogenesis [20-22]. For example, fibrin has been combined with ECM particles to develop implants for focal cartilage defect repair [23]. The use of such injectable hydrogels can also overcome limitations associated with pre-formed scaffolds, including challenges associated with fixation to complex cartilage defects and poor retention of newly synthesized ECM [24]. Therefore, such ECM functionalized fibrin hydrogels could potentially be used as an injectable carrier for freshly isolated stromal cells, with such a construct forming the basis of a single-stage therapy for articular cartilage regeneration. Hence, the objective of this study was to functionalize fibrin hydrogels with particulated cartilage ECM, and to assess the capacity of this construct to promote chondrogenesis of freshly isolated stromal cells in vivo. As promoting robust chondrogenesis in vivo may also necessitate exogenous growth factor presentation, this study also assessed the capacity of particulated cartilage ECM components to act as a controlled delivery system for TGF- $\beta 3$ within a fibrin hydrogel. The chondro-inductivity of these cartilage ECM components was then compared to gelatin microspheres. These specific strategies were evaluated both in vitro and in vivo with the aim of assessing their potential as putative single-stage therapies for cartilage repair. 


\section{Material and methods}

\subsection{Preparation of particulated cartilage ECM}

Articular cartilage for the fabrication of ECM particles was obtained from the articular joint of pigs (female, 3 months old), as presented in published work [12]. Briefly, cartilage was fragmented, and further fine ECM microparticles were fabricated by pulverizing/devitalizing the cartilage ECM in pieces with a cryogenic mill (6770 Freezer-Mill, SPEX). The ECM-derived particles were lyophilized (FreeZone-Triad, Labconco, USA), physically crosslinked and sterilized overnight using dehydrothermal treatment at $110^{\circ} \mathrm{C}$ under vacuum.

\subsection{SEM imaging of ECM-derived particles}

ECM microparticles were observed and analyzed using scanning electron microscopy (SEM). Particles were fixed in $4 \%$ paraformaldehyde solution overnight. Microparticles were dehydrated through successive graded ethanol baths (10-100\%), fixed in aluminium stubs, coated with gold and examined under a field emission scanning electron microscope (Tescan Mira FEG-SEM XMU, Libušina, Czech Republic). Images were analyzed with Image $\mathrm{J}$ to quantify microparticle size.

\subsection{Fabrication of Fibrin/ECM hydrogels}

Fibrin hydrogels were produced using a method previously described [14, 15]. Briefly, fibrin hydrogel constructs were fabricated by dissolving $100 \mathrm{mg} / \mathrm{ml}$ bovine fibrinogen (SigmaAldrich) in 10,000 KIU/ml aprotinin (Nordic Pharma, Sweden) containing $19 \mathrm{mg} / \mathrm{ml}$ sodium chloride $(\mathrm{NaCl})$. Solution of Thrombin $(5 \mathrm{U} / \mathrm{ml})$ was made in $40 \mathrm{mM}$ calcium chloride and adjusted to $\mathrm{pH}$ 7.0. Moreover, the optimal percentage loading of ECM particles in fibrin constructs was assessed based on literature $[21,25]$ and additional experimental work. ECM was mixed with fibrinogen (fibrin) solution in $2 \%(\mathrm{w} / \mathrm{v})$ and $10 \%(\mathrm{w} / \mathrm{v})$ based on similar approaches [21, 25]. An additional fibrin only was also prepared to serve as a control group. Fibrin/ECM or fibrin only solutions were mixed at a ratio of $1: 1$ with a $5 \mathrm{U} / \mathrm{ml}$ thrombin in 40 
$\mathrm{mM} \mathrm{CaCl}$ solution and allowed to gel at $37^{\circ} \mathrm{C}$ for 30 minutes yielding a final concentration of $50 \mathrm{mg} / \mathrm{ml}$ fibrin, $2.5 \mathrm{U} / \mathrm{ml}$ thrombin, $5,000 \mathrm{KIU} / \mathrm{ml}$ aprotinin, $17 \mathrm{mg} / \mathrm{ml} \mathrm{NaCl}$ and $20 \mathrm{mM} \mathrm{CaCl}{ }_{2}$ $[14,15]$. The final acellular hydrogels were $60 \mu \mathrm{l}$ and were produced by using cylindrical agarose moulds ( $3 \% \mathrm{w} / \mathrm{v}$; Sigma-Aldrich, Ireland), $5 \mathrm{~mm}$ in diameter. Furthermore, these fibrin/ECM hydrogels were scaled up for assessing the scalability of the method. The fibrin only, fibrin/ECM $2 \%$ and $10 \%(\mathrm{w} / \mathrm{v})$ acellular hydrogels were prepared by using a $1.5 \mathrm{ml}$ eppendorf tube as a mould.

\subsection{Fabrication of Gelatin Microspheres and Fibrin/Gelatin hydrogels}

Fibrin/gelatin hydrogels were produced using a previously described protocol [14], using a method similar to fibrin/ECM. However, in this particular case, gelatin microspheres $[14,26,27]$ were used and incorporated into the fibrin hydrogel as previously described [14]. Briefly, microspheres were produced by a water-in-oil emulsion method. Gelatin was dissolved in deionised water and added drop-by-drop to $100 \mathrm{ml}$ of olive oil heated to $45^{\circ} \mathrm{C}$ while being continuously stirred. Gelatin concentration of $11 \%(w / v)$ was used in this study. After 10 minutes, the solution was cooled with additional stirring for 30 minutes, after which $40 \mathrm{ml}$ of acetone was added and left for 1 hour. Formed gelatin microspheres were collected through sieving $(50 \mu \mathrm{m})$ and repeated washings in acetone. Microspheres were next crosslinked in $100 \mathrm{ml}$ of glutaraldehyde solution (0.1\% w/v; Sigma-Aldrich, Ireland) with 100 $\mu$ Tween 80 (Sigma-Aldrich, Ireland) for 18 hours while being stirred. Then they were removed from the glutaraldehyde solution and stirred in $100 \mathrm{ml}$ of glycine solution $(25 \mathrm{mM}$, Sigma-Aldrich, Ireland) solution for 1 hour. Microspheres were sieved to a controlled range (50-70 $\mu \mathrm{m})$, which was used for previously reported release studies [14]. Finally, microspheres were freeze-dried overnight, weighed and sterilized using dehydrothermal treatment [14].

\subsection{Cell isolation and culture}

Cells were isolated from biopsies of the infrapatellar fat pad (IFP) taken from human patients either undergoing total joint arthroplasty (3 donors) or anterior cruciate ligament 
surgery (1 donor) with informed signed consent. Ethical approval for this work was granted by the Mater Misericordiae University Hospital (Ireland) Ethics Committee (Ref: 1/378/1501). IFP was minced and rotated at $37^{\circ} \mathrm{C}(4 \mathrm{~h})$ in Dulbecco's Modified Eagle Medium (hgDMEM, GlutaMAX ${ }^{\mathrm{TM}}$; Gibco) with collagenase (type 2, $750 \mathrm{U} / \mathrm{ml}$, Worthington Bio.) and penicillin (1\%, $100 \mathrm{U} / \mathrm{ml})$-streptomycin $(100 \mu \mathrm{g} / \mathrm{ml})$. Based on previous work, $4 \mathrm{ml} / \mathrm{g}$-tissue of collagenase was used $[28,29]$. Cells were expanded (5000 cells $/ \mathrm{cm}^{2}$; to P2) in hgDMEM with FBS (10\%), penicillin (1\%, $100 \mathrm{U} / \mathrm{ml})$-streptomycin $(100 \mathrm{mg} / \mathrm{ml})$, and FGF-2 $(5 \mathrm{ng} / \mathrm{ml}$; ProSpec., Israel).

Chondrogenesis was assessed in vitro by encapsulating 1 million IFP-derived stem cells from one donor into fibrin hydrogels $(60 \mu \mathrm{l})$. Hydrogel-cell culture (4 weeks) was performed in chondrogenic specific incubation conditions: $5 \% \mathrm{O}_{2}$ and $37^{\circ} \mathrm{C}[11,12,29]$. Briefly, the medium consisted of DMEM supplemented with penicillin $(100 \mathrm{U} / \mathrm{ml})$ streptomycin $(100 \mu \mathrm{g} / \mathrm{ml})$, sodium pyruvate $(100 \mu \mathrm{g} / \mathrm{ml})$, L-proline $(40 \mu \mathrm{g} / \mathrm{ml})$, ascorbic acid (50 $\mu \mathrm{g} / \mathrm{ml})$, bovine serum albumin (1.5 $\mathrm{mg} / \mathrm{ml})$, insulin-transferrin-selenium (1x), dexamethasone (100 nM), and TGF- $\beta 3$ (10 ng/ml, ProSpec., Israel). The medium of experimental groups where TGF- $\beta 3$ was added into the hydrogel were not additionally supplemented with this growth factor. The growth factor (200 ng) was soak-loaded into the ECM particles and gelatin microspheres for 15 minutes prior to hydrogel encapsulation.

\subsection{DNA, sulphated glycosaminoglycan (sGAG) and collagen content}

Before and after the 4 week culture period, standard biochemical analyses were performed to determine the DNA, SGAG and collagen content, based on previously described protocols $[11,12,28,30]$. Firstly, the cartilaginous tissue grafts were digested at $60^{\circ} \mathrm{C}$ for 18 hours in papain $(125 \mu \mathrm{g} / \mathrm{ml})$. DNA was determined via a Hoechst assay; sGAG in constructs was obtained using a dimethylmethylene blue based assay, and finally the collagen content was measured by determining the hydroxyproline content after acidic hydrolysis (chloramine-T with a hydroxyproline to collagen ratio of 1/7.69). The SGAG and collagen content at day 0 , which represents that contained within the ECM particles and 
gelatin microspheres themselves, is subtracted from the day 28 values for data presentation purposes.

\subsection{Histological and immunohistochemical analysis}

Histology analysis was performed as previously described [12]. Briefly, samples were fixed in $4 \%$ paraformaldehyde, followed by wax embedding and sectioning ( $6 \mu \mathrm{m})$. Sections were stained for sGAG using alcian blue and collagen with picro-sirius red. Cell nuclei were additionally stained with nuclear fast red solution (all reagents from Sigma-Aldrich). Immunohistochemical examination was performed using a monoclonal antibody to type II collagen (Abcam, UK), as previously described [11, 12, 29].

\subsection{Growth factor measurement}

TGF- $\beta 3$ released from fibrin/ECM and fibrin/gelatin constructs was quantified through an enzyme-linked immunosorbent assay (ELISA) protocol, as previously described [11, 12, 32]. Briefly, well-plates were firstly coated with capture antibody (mouse anti-human TGF$\beta 3)$, followed by culture media incubation. Next, the detection antibody was supplemented to each well. Streptavidin-HRP was then incubated, followed by substrate solution. Finally, the stop solution was used, and the optical measurement was performed (all reagents from ELISA kit, R\&D Systems).

\subsection{Subcutaneous implantation assessment}

For the first in vivo assessment, fibrin/ECM hydrogels with and without $200 \mathrm{ng}$ of TGF- $\beta 3$, were seeded with freshly isolated IFP derived stromal cells and then implanted subcutaneously in nude mice, as previously described [12]. The second in vivo study compared the following groups (where all constructs were loaded with 200 ng of TGF- $\beta 3$ ): 1. Acellular Fibrin/Gelatin; 2. Stromal cells in Fibrin/Gelatin; 3. Acellular Fibrin/ECM; 4. Stromal cells in Fibrin/ECM. Freshly isolated IFP-derived stromal cells were pooled from 3 donors. Cells were not culture expanded prior to implantation. The animal protocol was reviewed and approved by Trinity College Dublin animal research ethics committee (Ref: 231112). 
Anaesthesia was performed with xylazine (10 mg/kg, Chanazine $2 \%$, Chanelle) and ketamine (100 mg/kg, Narketan, Vetoquinol). Incision and construct insertion was performed along the spine, and sutures were made with 4-0 Vicryl plus (Ethicon, Johnson \& Johnson) followed by tissue glue (Vetloc).

\subsection{Statistics}

All the results are reported as mean \pm standard deviation. Analyses were performed using MINITAB 15.1 (Minitab, UK). Groups were analyzed for significant differences using a linear model of variance analysis (ANOVA) with factors including TGF- $\beta 3$ supplementation and hydrogel composition. Tukey's test for comparisons was performed, with significance accepted for $p<0.05$.

\section{Result}

\subsection{Development of stable ECM functionalized fibrin hydrogels}

Scanning electron microscopy (SEM) was used to characterize the morphology and size distribution of the particulated ECM (Figure $1 \mathrm{~A}-\mathrm{C}$ ), with the mean particle size after cryomilling measured as $97 \pm 26 \mu \mathrm{m}$. Fibrin was then mixed with particulated ECM material at two different concentrations. To assess if the incorporation of different concentrations of ECM would impact the capacity of fibrin to form a stable gel, the components were added to a cylindrical mould (Figure 1D-F) and an eppendorf tube (Figure 1G, H) and their capacity to maintain their shape was determined. The ECM free fibrin hydrogel appeared semitransparent (Figure 1D) and with the addition of ECM became more opaque (Figure 1E, F). The capacity of the hydrogels to maintain a fixed shape also diminished with the incorporation of higher concentrations of ECM, as it was possible to observe a stable structure for fibrin only (data not shown) and fibrin/ECM 2\% w/v (Figure 1G); however when the ECM concentration was increased $(10 \% \mathrm{w} / \mathrm{v})$ the structure failed to maintain its initial 
shape (Figure $1 \mathrm{H})$. This motivated the use of $2 \%(\mathrm{w} / \mathrm{v})$ ECM embedded in fibrin hydrogel for further assessment.

\subsection{Fibrin hydrogels functionalized with particulated cartilage ECM support robust chondrogenesis when stimulated with TGF- $\beta 3$}

We next sought to determine the capacity of fibrin hydrogels functionalized with cartilage ECM to support chondrogenesis of infrapatellar fat pad-derived stem cells in vitro in the presence and absence of TGF- $\beta 3$. After 28 days of in vitro culture, fibrin-ECM composites supplemented with TGF- $\beta 3$ resembled cartilage macroscopically (Figure 2E). When compared with growth factor free constructs (Figure 2A-D), the TGF- $\beta 3$ supplemented hydrogels (Figure 2E-H) contained higher levels of cartilage specific matrix components. Histological staining for sGAG (Figure 2F), collagen (Figure 2G) and type II collagen (Figure $2 \mathrm{H})$ was more intense for TGF- $\beta 3$ supplemented constructs. The DNA, sGAG and collagen content of TGF- $\beta 3$ supplemented hydrogels was significantly higher than non-supplemented constructs (Figure $2 \mathrm{I}-\mathrm{K}$ ).

\subsection{ECM-derived particles can be used to deliver TGF- $\beta 3$ and enhance chondrogenesis of human infrapatellar fat pad-derived stem cells}

Having demonstrated superior chondrogenesis in the presence of TGF- $\beta 3$, and with a view towards developing an "off-the-shelf" therapeutic for cartilage regeneration, we next sought to assess the potential of particulated cartilage ECM to bind and release TGF- $\beta 3$ within a stem cell laden hydrogel, and to subsequently support chondrogenesis over 28 days of in vitro culture. Furthermore, we sought to determine if the incorporation of ECM into fibrin would enhance chondrogenesis compared to that within control fibrin hydrogels containing gelatin microspheres. After loading the ECM-derived particles with TGF- $\beta 3$ it was possible to observe a progressive release of the growth factor into the surrounding culture (Figure 3), comparable to that obtained using gelatin microspheres embedded in the same stem cellladen fibrin hydrogel (Figure 3). 
At the end of the 28 day in vitro culture period, both types of constructs resembled cartilage macroscopically (Figure 4A, E). Histologically, the tissue generated with the ECM functionalized hydrogels was richer in sGAGs and type II collagen (Figure 4B-D). In addition, significantly higher levels of sGAG (Figure 4J) and collagen (Figure 4K) accumulation were observed with ECM functionalized constructs. No significant difference in DNA content was observed between the two groups (Figure 4I).

\subsection{Cartilage ECM enhances chondrogenesis in vivo in the presence of TGF- $\beta 3$}

It was pertinent to determine the potential of fibrin hydrogels functionalized with particulated cartilage ECM hydrogels to promote chondrogenesis in vivo in a subcutaneous nude mouse model when seeded with freshly isolated infrapatellar fat pad-derived stromal cells. The particulated ECM was either loaded with TGF- $\beta 3$ (+TGF- $\beta 3$ ) or left empty (-TGFß3) prior to implantation. After 4 weeks in vivo the Fibrin-ECM constructs loaded with TGF33 generated more robust chondrogenesis of human infrapatellar fat pad stromal cells, with superior sGAG (Figure 5F), collagen (Figure 5G) and type II collagen (Figure 5H) deposition compared to the non-supplemented group (Figure 5B-D).

The final goal of the study was to determine whether particulated cartilage ECM is chondroinductive in vivo. To this end, the capacity of fibrin hydrogels functionalized with either gelatin microspheres or particulated cartilage ECM to promote ectopic cartilage formation was compared. Both the gelatin and cartilage ECM particles were loaded with TGF- $\beta 3$ and the constructs were either implanted cell free or with freshly isolated stromal cells. Little matrix appeared to accumulate within acellular constructs after 4 weeks in vivo (Figure 6). Macroscopically, ECM constructs seeded with freshly isolated stromal cells appeared cartilage-like in appearance (Figure 6M), unlike the other fibrin based groups. More intense staining for sGAG (Figure 6N), collagen (Figure 6O) and type II collagen (Figure 6P) deposition was observed in fibrin-ECM constructs when compared with the fibrin-gelatin constructs. sGAG synthesis in the fibrin-ECM implants was significantly higher than in the fibrin-gelatin constructs (Figure 6Q). 


\section{Discussion}

The goal for this study was to develop an injectable, single-stage strategy to promote chondrogenesis in vivo. The method consists of functionalizing a well-established biomaterial (fibrin) with particulated cartilage ECM loaded with TGF- $\beta 3$, and using this construct to promote chondrogenesis of freshly isolated IFP-derived stromal cells. By cryomilling and freeze-drying the ECM it was possible to produce microparticles that were easily incorporated into fibrin hydrogels at a known concentration. In spite of the fact that these particles are potentially chondroinductive on their own, due to the sGAGs, collagen and innate chondrogenic cues within the ECM, additional TGF- $\beta 3$ stimulation was necessary to induce robust chondrogenesis both in vitro and in vivo. In vitro, ECM-derived particles were able to retain and release TGF- $\beta 3$, proving to be as effective in this regard as the well established gelatin microsphere delivery system [14]. Fibrin hydrogels functionalized with particulated cartilage ECM promoted superior chondrogenesis in vitro and in vivo when compared with fibrin-gelatin constructs. These findings open up the possibility of using ECM functionalized fibrin hydrogels, in combination with freshly isolated stromal cells, to regenerate articular cartilage defects.

Cryomilling and freeze-drying of cartilage ECM was found to result in the development of particulated tissue with a consistent size and morphology, with comparable strategies being employed in the literature [33]. While we demonstrated that the specific size of ECM particles used in this study enhanced chondrogenesis in vitro and in vivo, further studies need to be performed to understand the role of ECM particle size in promoting chondrogenesis using this approach. Previous studies have demonstrated that the particle size of powdered ECM material can influence new tissue formation, regardless of initial biomaterial composition [34]. Furthermore, particle size will also determine the effectiveness of devitalization and decellularization treatments that will likely influence the immune response [35]. Additional studies need to be undertaken to better understand the immunogenicity of cartilage ECM-derived materials, and consequently understand and 
optimize production and decellularization methods to enable clinical translation of such powerful naturally-derived biomaterials [35].

In agreement with previous studies [11, 12], additional growth factor (TGF- $\beta 3$ ) stimulation was necessary to induce robust chondrogenesis of IFP-derived stem cells, even in the presence of particulated cartilage ECM. This motivated us to explore the use of cartilage ECM particles as a growth factor delivery system. These ECM particles were comparable to traditional gelatin microspheres in their capacity to bind and release TGF- $\beta 3$. It is well known that ECM components can act as reservoirs for efficient growth factor release $[11,12,36,37]$. Proteoglycans (negatively charged) present in the pericellular and extracellular matrix have been shown to bind and modulate TGF- $\beta 3$ (positively charged) supply and consequently control its availability [36].

While the amount and rate of TGF- $\beta 3$ release from cartilage ECM was similar to that from gelatin microspheres, superior chondrogenesis was observed in the ECMfunctionalized hydrogels. This difference indicates that the ECM-derived particles are able to provide additional chondroinductive cues to the IFP-derived stem cells that are not present in the fibrin/gelatin hydrogels, strongly suggesting that these bioactive ECM microparticles are more than simple growth factor carriers. The presence of growth factors in cartilage ECM, particularly from the TGF- $\beta$ superfamily, may explain the superior chondrogenesis observed in these constructs $[18,35,38-40]$. These growth factors can bind and be stored in many of the matrix molecules present in native cartilage ECM [38]. Additionally, the presence of type II collagen in cartilage ECM is known to be advantageous for chondrogenesis [41-44], which likely also contributes to the superior results observed in the cartilage ECM-functionalized biomaterials.

Growth factor release from the hydrogel to the media was less than $10 \%$ of the initial amount loaded into the microparticles. Fibrin by itself has been used previously as a growth factor delivery system $[13,14]$, and hence it is likely helping to retain the TGF- $\beta 3$ within the construct. Results from ELISA indicate that the release of growth factor from particles occurs at a relatively slow rate throughout the culture period, except for a more rapid release profile 
during the first 8 days. It is not clear what percentage of growth factor that remains within the construct is available to seeded cells. Regardless, this specific release profile is sufficient to initiate the chondrogenic pathway. Temporal growth factor exposure is known to be beneficial in chondrogenesis [11, 12, 45-47]. Further studies are necessary to identify the optimal amount of TGF- $\beta 3$ that needs to be added to the delivery device in order to promote robust chondrogenesis of embedded cells. Previous studies suggest that only relatively low levels of growth factor need to be added to ECM-based hydrogels or scaffolds to enhance cartilage formation $[11,12,35,39,48,49]$.

ECM functionalized fibrin was successfully used as a vehicle to subcutaneously deliver freshly isolated IFP-derived stromal cells and growth factor. The devitalized cartilage ECM-derived particles facilitated robust chondrogenesis of the encapsulated stromal cells in this in vivo environment. These stromal cells were critical to generate cartilage ectopically, as acellular fibrin/ECM constructs did not induce chondrogenesis. When compared with the well established gelatin microspheres system, the ECM particles induced superior cartilage matrix formation, emphasizing the important role and potential of such particles in vivo.

Greater differences in chondrogenesis were observed between the fibrin/ECM constructs and fibrin/gelatin constructs in vivo than were observed in vitro. Such differences may be due, at least in part, to the different cell populations used in the in vitro and in vivo studies. Culture expanded infrapatellar fat pad-derived stem cells were used for the in vitro studies, while freshly isolated stromal cells isolated from the infrapatellar fat pad (i.e. no culture expansion prior to implantation) were used for the in vivo studies. We typically observe poor chondrogenesis using freshly isolated stromal cells in vitro, therefore we typically use culture expanded stem cells for evaluating different hydrogel/scaffold designs in vitro. The culture expanded stem cells likely have a stronger overall chondrogenic potential; hence generating relatively robust chondrogenesis in vitro in both the fibrin/ECM and fibrin/gelatin constructs. We speculate that the freshly isolated stromal cell population is more responsive to the cues provided by the cartilage ECM components. In addition, 
uncharacterized differences between the in vitro culture environment and that in the back of a mouse are also likely playing a role.

Finally, it is relevant to affirm that further studies need to be performed to assess immune response, alternative methods of devitalization and decellularization, and the capacity of the proposed therapy to regenerate cartilage defects in large animal models. From a translation perspective, the proposed strategy of freshly isolating fat pad stromal cells and embedding them in an ECM functionalized fibrin hydrogel seems promising, as it may be used as a minimally invasive approach for single-stage cartilage repair. The strategy of cryomilling and devitalizing native ECM to produce bioactive particles, and incorporating them in a well-established fibrin hydrogel with stromal cells and/or growth factors, has multiple potential applications for tissue regeneration.

\section{Conclusion}

The objective of this study was to functionalize an injectable fibrin hydrogel with a view to developing a single-stage therapy for cartilage repair. We demonstrate that ECMderived microparticles can deliver TGF- $\beta 3$ and that this system can induce chondrogenesis of freshly isolated fat pad-derived stromal cells in vivo. This finding supports the concept that populations of freshly isolated stromal cells, when combined with a chondroinductive hydrogel, can induce cartilage formation and can potentially be used in single-stage, minimally invasive procedures for cartilage regeneration. The translation of such a strategy would overcome many of the current limitations associated with clinically available cellbased therapies for cartilage repair.

\section{Acknowledgments}

Funding was provided by a European Research Council Starter Grant (StemRepair Project number: 258463), Programme for Research in Third-Level Institutions (PRTLI) Graduate Research Education Programme in Engineering, and co-funded by the European Regional Development Fund and the HEA. 


\section{References}

[1] Madeira C, Santhagunam A, Salgueiro JB, Cabral JM. Advanced cell therapies for articular cartilage regeneration. Trends Biotechnol. 2015;33:35-42.

[2] Felson DT. Osteoarthritis of the Knee. New England Journal of Medicine. 2006;354:8418.

[3] Brittberg M. Autologous chondrocyte implantation--technique and long-term follow-up. Injury. 2008;39 Suppl 1:S40-9.

[4] Gobbi A, Karnatzikos G, Sankineani SR. One-step surgery with multipotent stem cells for the treatment of large full-thickness chondral defects of the knee. The American journal of sports medicine. 2014;42:648-57.

[5] Jurgens WJFM, van Dijk A, Doulabi BZ, Niessen FB, Ritt MJPF, van Milligen FJ, et al. Freshly isolated stromal cells from the infrapatellar fat pad are suitable for a one-step surgical procedure to regenerate cartilage tissue. Cytotherapy. 2009;11:1052-64.

[6] Kon E, Filardo G, Roffi A, Andriolo L, Marcacci M. New trends for knee cartilage regeneration: from cell-free scaffolds to mesenchymal stem cells. Curr Rev Musculoskelet Med. 2012;5:236-43.

[7] Bekkers JE, Creemers LB, Tsuchida Al, van Rijen MH, Custers RJ, Dhert WJ, et al. Onestage focal cartilage defect treatment with bone marrow mononuclear cells and chondrocytes leads to better macroscopic cartilage regeneration compared to microfracture in goats. Osteoarthritis and cartilage / OARS, Osteoarthritis Research Society. 2013;21:9506.

[8] Bekkers JE, Tsuchida AI, van Rijen MH, Vonk LA, Dhert WJ, Creemers LB, et al. Singlestage cell-based cartilage regeneration using a combination of chondrons and mesenchymal stromal cells: comparison with microfracture. The American journal of sports medicine. 2013;41:2158-66. 
[9] Liu Y, Buckley CT, Downey R, Mulhall KJ, Kelly DJ. The role of environmental factors in regulating the development of cartilaginous grafts engineered using osteoarthritic human infrapatellar fat pad-derived stem cells. Tissue engineering Part A. 2012;18:1531-41.

[10] Liu Y, Buckley CT, Almeida H, Mulhall K, Kelly DJ. Infrapatellar Fat Pad Derived Stem Cells Maintain Their Chondrogenic Capacity in Disease and can be used to Engineer Cartilaginous Grafts of Clinically Relevant Dimensions. Tissue engineering Part A. 2014.

[11] Almeida HV, Liu Y, Cunniffe GM, Mulhall KJ, Matsiko A, Buckley CT, et al. Controlled release of transforming growth factor- $\beta 3$ from cartilage-extra-cellular-matrix-derived scaffolds to promote chondrogenesis of human-joint-tissue-derived stem cells. Acta biomaterialia. 2014.

[12] Almeida HV, Cunniffe GM, Vinardell T, Buckley CT, O'Brien FJ, Kelly DJ. Coupling Freshly Isolated CD44+ Infrapatellar Fat Pad-Derived Stromal Cells with a TGF- $\beta 3$ Eluting Cartilage ECM-Derived Scaffold as a Single-Stage Strategy for Promoting Chondrogenesis. Advanced Healthcare Materials. 2015;4:1043-53.

[13] Spicer PP, Mikos AG. Fibrin glue as a drug delivery system. Journal of controlled release : official journal of the Controlled Release Society. 2010;148:49-55.

[14] Ahearne M, Buckley CT, Kelly DJ. A growth factor delivery system for chondrogenic induction of infrapatellar fat pad-derived stem cells in fibrin hydrogels. Biotechnology and Applied Biochemistry. 2011;58:345-52.

[15] Sheehy EJ, Mesallati T, Vinardell T, Kelly DJ. Engineering cartilage or endochondral bone: A comparison of different naturally derived hydrogels. Acta biomaterialia. 2015;13:245-53.

[16] Thorpe SD, Buckley CT, Steward AJ, Kelly DJ. European Society of Biomechanics S.M. Perren Award 2012: The external mechanical environment can override the influence of local substrate in determining stem cell fate. Journal of Biomechanics. 2012;45:2483-92.

[17] Im G-I. Chondrogenesis from mesenchymal stem cells derived from adipose tissue on the fibrin scaffold. Current Applied Physics. 2005;5:438-43. 
[18] Benders KEM, Weeren PRv, Badylak SF, Saris DBF, Dhert WJA, Malda J. Extracellular matrix scaffolds for cartilage and bone regeneration. Trends in Biotechnology. 2013;31:16976.

[19] Rowland CR, Lennon DP, Caplan AI, Guilak F. The effects of crosslinking of scaffolds engineered from cartilage ECM on the chondrogenic differentiation of MSCs. Biomaterials. 2013;34:5802-12.

[20] Visser J, Gawlitta D, Benders KE, Toma SM, Pouran B, van Weeren PR, et al. Endochondral bone formation in gelatin methacrylamide hydrogel with embedded cartilagederived matrix particles. Biomaterials. 2015;37:174-82.

[21] Chang C-H, Chen C-C, Liao C-H, Lin F-H, Hsu Y-M, Fang H-W. Human acellular cartilage matrix powders as a biological scaffold for cartilage tissue engineering with synovium-derived mesenchymal stem cells. Journal of Biomedical Materials Research Part A. $2013 ; 102(7): 2248-57$.

[22] Sutherland AJ, Detamore MS. Bioactive Microsphere-Based Scaffolds Containing Decellularized Cartilage. Macromol Biosci. 2015;15:979-89.

[23] Abrams GD, Mall NA, Fortier LA, Roller BL, Cole BJ. BioCartilage: Background and Operative Technique. Operative Techniques in Sports Medicine. 2013;21:116-24.

[24] Grad S, Kupcsik L, Gorna K, Gogolewski S, Alini M. The use of biodegradable polyurethane scaffolds for cartilage tissue engineering: potential and limitations. Biomaterials. 2003;24:5163-71.

[25] Kwon JS, Yoon SM, Shim SW, Park JH, Min KJ, Oh HJ, et al. Injectable extracellular matrix hydrogel developed using porcine articular cartilage. International journal of pharmaceutics. 2013;454:183-91.

[26] Holland TA, Tabata Y, Mikos AG. In vitro release of transforming growth factor- $\beta 1$ from gelatin microparticles encapsulated in biodegradable, injectable oligo(poly(ethylene glycol) fumarate) hydrogels. Journal of Controlled Release. 2003;91:299-313. 
[27] Park H, Temenoff JS, Holland TA, Tabata Y, Mikos AG. Delivery of TGF- $\beta 1$ and chondrocytes via injectable, biodegradable hydrogels for cartilage tissue engineering applications. Biomaterials. 2005;26:7095-103.

[28] Ignat'eva NY, Danilov NA, Averkiev SV, Obrezkova MV, Lunin VV, Sobol EN. Determination of hydroxyproline in tissues and the evaluation of the collagen content of the tissues. Journal of Analytical Chemistry. 2007;62:51-7.

[29] Buckley CT, Kelly DJ. Expansion in the presence of FGF-2 enhances the functional development of cartilaginous tissues engineered using infrapatellar fat pad derived MSCs. Journal of the mechanical behavior of biomedical materials. 2012;11:102-11.

[30] Guillaume O, Daly A, Lennon K, Gansau J, Buckley SF, Buckley CT. Shape-memory porous alginate scaffolds for regeneration of the annulus fibrosus: effect of TGF-beta3 supplementation and oxygen culture conditions. Acta biomaterialia. 2014;10:1985-95.

[31] Buckley CT, Meyer EG, Kelly DJ. The influence of construct scale on the composition and functional properties of cartilaginous tissues engineered using bone marrow-derived mesenchymal stem cells. Tissue Engineering - Part A. 2012;18:382-96.

[32] Vonwil D, Wendt D, Ströbel S, Wallny HJ, Gygax D, Heberer M, et al. Assessment of the stability of TGFß3 bioactivity for potential bioreactor applications. Biochemical Engineering Journal. 2008;39:586-9.

[33] Gilbert TW, Stolz DB, Biancaniello F, Simmons-Byrd A, Badylak SF. Production and characterization of ECM powder: implications for tissue engineering applications. Biomaterials. 2005;26:1431-5.

[34] Sampath TK, Reddi AH. Importance of geometry of the extracellular matrix in endochondral bone differentiation. The Journal of cell biology. 1984;98:2192-7.

[35] Sutherland AJ, Converse GL, Hopkins RA, Detamore MS. The Bioactivity of Cartilage Extracellular Matrix in Articular Cartilage Regeneration. Advanced Healthcare Materials. 2015;4:29-39.

[36] Macri L, Silverstein D, Clark RAF. Growth factor binding to the pericellular matrix and its importance in tissue engineering. Advanced Drug Delivery Reviews. 2007;59:1366-81. 
[37] Holland TA, Mikos AG. Advances in drug delivery for articular cartilage. Journal of Controlled Release. 2003;86:1-14.

[38] Albro MB, Nims RJ, Cigan AD, Yeroushalmi KJ, Alliston T, Hung CT, et al. Accumulation of exogenous activated TGF-beta in the superficial zone of articular cartilage. Biophysical journal. 2013;104:1794-804.

[39] Cheng NC, Estes BT, Awad HA, Guilak F. Chondrogenic differentiation of adiposederived adult stem cells by a porous scaffold derived from native articular cartilage extracellular matrix. Tissue engineering Part A. 2009;15:231-41.

[40] Cheng CW, Solorio LD, Alsberg E. Decellularized tissue and cell-derived extracellular matrices as scaffolds for orthopaedic tissue engineering. Biotechnology advances. $2014 ; 32: 462-84$.

[41] Bosnakovski D, Mizuno M, Kim G, Takagi S, Okumura M, Fujinaga T. Chondrogenic differentiation of bovine bone marrow mesenchymal stem cells (MSCs) in different hydrogels: influence of collagen type II extracellular matrix on MSC chondrogenesis. Biotechnol Bioeng. 2006;93:1152-63.

[42] Rutgers M, Saris DB, Vonk LA, van Rijen MH, Akrum V, Langeveld D, et al. Effect of collagen type I or type II on chondrogenesis by cultured human articular chondrocytes. Tissue engineering Part A. 2013;19:59-65.

[43] Ragetly G, Griffon DJ, Chung YS. The effect of type II collagen coating of chitosan fibrous scaffolds on mesenchymal stem cell adhesion and chondrogenesis. Acta biomaterialia. 2010;6:3988-97.

[44] Ragetly GR, Griffon DJ, Lee HB, Chung YS. Effect of collagen II coating on mesenchymal stem cell adhesion on chitosan and on reacetylated chitosan fibrous scaffolds. Journal of materials science Materials in medicine. 2010;21:2479-90.

[45] Buxton AN, Bahney CS, Yoo JU, Johnstone B. Temporal exposure to chondrogenic factors modulates human mesenchymal stem cell chondrogenesis in hydrogels. Tissue engineering Part A. 2011;17:371-80. 
[46] Byers BA, Mauck RL, Chiang IE, Tuan RS. Transient exposure to transforming growth factor beta 3 under serum-free conditions enhances the biomechanical and biochemical maturation of tissue-engineered cartilage. Tissue engineering Part A. 2008;14:1821-34.

[47] Chung C, Burdick JA. Influence of three-dimensional hyaluronic acid microenvironments on mesenchymal stem cell chondrogenesis. Tissue engineering Part A. 2009;15:243-54.

[48] Cheng NC, Estes BT, Young TH, Guilak F. Engineered cartilage using primary chondrocytes cultured in a porous cartilage-derived matrix. Regen Med. 2011;6:81-93.

[49] Diekman BO, Estes BT, Guilak F. The effects of BMP6 overexpression on adipose stem cell chondrogenesis: Interactions with dexamethasone and exogenous growth factors. Journal of biomedical materials research Part A. 2010;93:994-1003.

\section{Figure captions}

Figure 1 - Fibrin hydrogels functionalized with ECM microparticles. Cartilage before cryomilling (A). SEM micrograph of the pulverized ECM (B) and high magnification for cartilage particles (C) (scale bar: $500 \mu \mathrm{m}(\mathrm{B})$ and $100 \mu \mathrm{m}(\mathrm{C})$ ). Macroscopic appearance of cylindrically-shaped hydrogels: fibrin only (D), fibrin/ECM 2\% (w/v) (E) and fibrin/ECM 10\% w/v (F) (scale bar: $2 \mathrm{~mm}$ ). Alternative scaled-up geometry for fibrin/ECM 2\% (w/v) (G) and fibrin/ECM 10\% w/v (H).

Figure 2 - TGF- $\beta 3$ stimulation enhances chondrogenesis in fibrin/ECM hydrogels in vitro. Macroscopic view of unstimulated fibrin/ECM constructs $(A)$ and fibrin/ECM constructs stimulated with TGF- $\beta 3(E)$. All hydrogels are seeded with human fat pad-derived stem cells and maintained in culture for 28 days prior to analysis $\left(n=4,{ }^{*} p<0.05\right)$. Alcian blue $(A B)$, picro-sirius red (PR) and type II collagen (Coll II) staining for fibrin/ECM constructs (B-D) and fibrin/ECM constructs stimulated with TGF- $\beta 3$ hydrogel (F-H). DNA (I), sGAG (J) and collagen (K) accumulation within fibrin/ECM constructs (-TGF- $\beta 3$ ) and fibrin/ECM constructs stimulated with TGF- $\beta 3$ (+TGF- $\beta 3)$. Scale bar: $50 \mu \mathrm{m}$.

Figure 3 - Release of TGF- $\beta 3$ from ECM microparticles. TGF- $\beta 3$ release into the media from the fibrin/ECM constructs and fibrin/gelatin constructs, as measured by ELISA $(n=3)$. 
Cumulative release values are presented as a percentage of the initial amount of TGF- $\beta 3$ loaded into the scaffold.

Figure 4 - Chondrogenesis within TGF- $\beta 3$ releasing hydrogels in vitro. Macroscopic view of fibrin/ECM constructs loaded with TGF- $\beta 3$ (A) and fibrin/gelatin constructs loaded with TGF- $\beta 3$ (E). Alcian blue (AB), picro-sirius red (PR) and type II collagen (Coll II) staining for fibrin/ECM constructs loaded with TGF- $\beta 3$ (B-D) and fibrin/gelatin constructs loaded with TGF-ß3 (F-H). DNA (I), sGAG (J) and collagen (K) accumulation within fibrin/ECM constructs loaded with TGF- $\beta 3$ and fibrin/gelatin constructs loaded with TGF- $\beta 3$. All hydrogels were seeded with human fat pad-derived stem cells and maintained in culture for 28 days prior to analysis $\left(n=4,{ }^{*} p<0.05\right)$. Scale bar: $50 \mu$.

Figure 5 - TGF- $\beta 3$ delivery enhances chondrogenesis within ECM functionalized fibrin hydrogels in vivo. Macroscopic view of fibrin/ECM hydrogels without $(A)$ and with $(E)$ the incorporation of TGF- $\beta 3$. Alcian blue (AB), picro-sirius red (PR) and type II collagen (Coll II) staining of fibrin/ECM hydrogels without (B-D) and with $(F-H)$ the incorporation of TGF- $\beta 3$. Superior matrix deposition was observed in vivo when TGF- $\beta 3$ was soak-loaded into the ECM microparticles. All data corresponds to fibrin/ECM hydrogels after 28 days in vivo. Scale bar: $50 \mu \mathrm{m}$.

Figure 6 - Comparison of the capacity of fibrin/gelatin and fibrin/ECM hydrogels to support chondrogenesis in vivo. Macroscopic view of acellular fibrin/gelatin (A), acellular fibrin/ECM (I), fibrin/gelatin seeded with freshly isolated stromal cells (E) and fibrin/ECM seeded with freshly isolated stromal cells (M). All constructs were loaded with TGF- $\beta 3$ prior to implantation. Alcian blue (AB), picro-sirius red (PR) and type II collagen (Coll II) staining for acellular (B-D) and cell-laden (F-H) fibrin/gelatin constructs and acellular (J-L) and cellladen (N-P) fibrin/ECM constructs. sGAG/DNA accumulation within cell-laden fibrin/ECM constructs compared to fibrin/gelatin constructs $(Q)\left(n=6,{ }^{*} p<0.05\right)$. All data corresponds to constructs implanted for 28 days in vivo. Scale bar: $50 \mu \mathrm{m}$. 
Fig 1

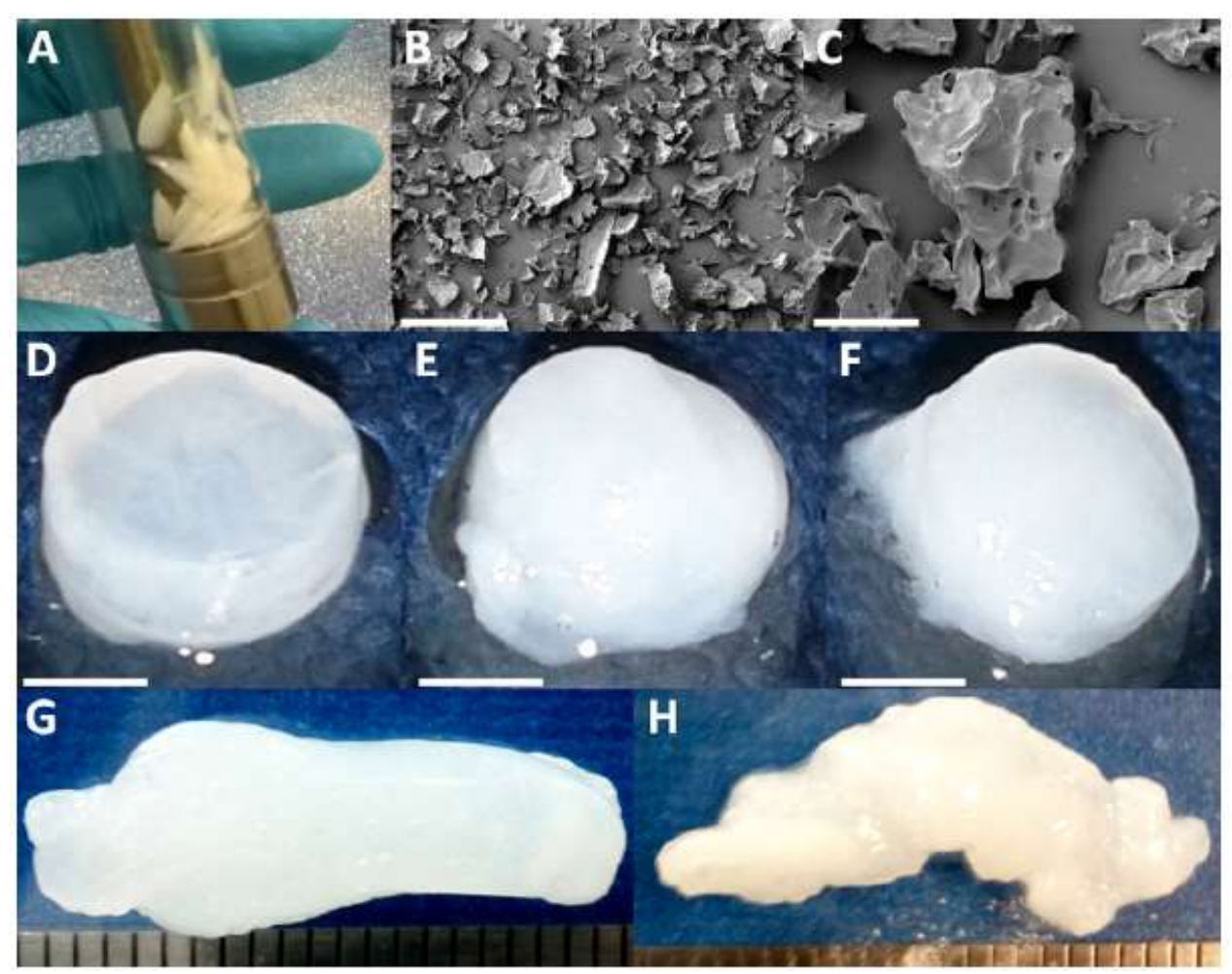

Fig 2

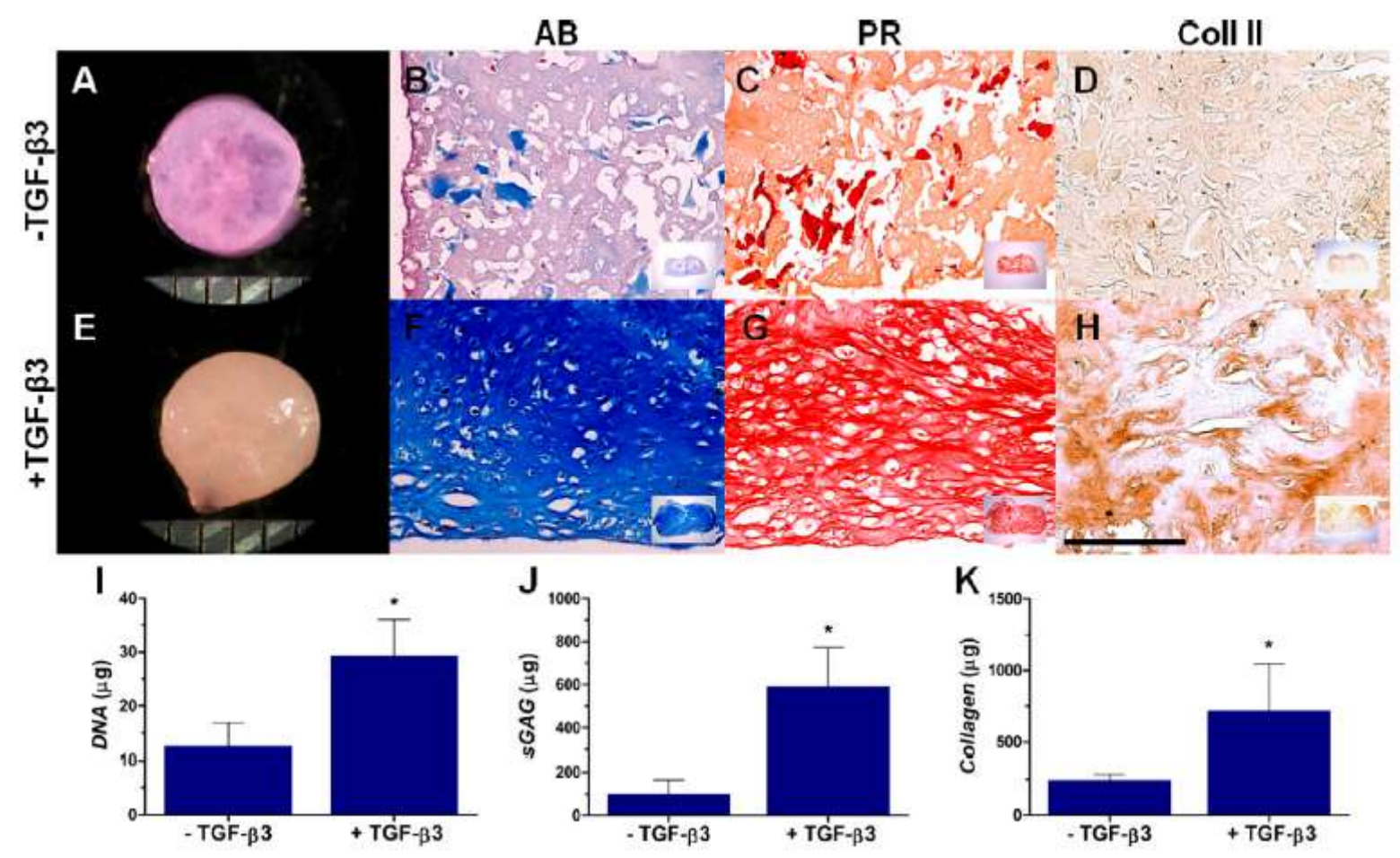


Fig 3

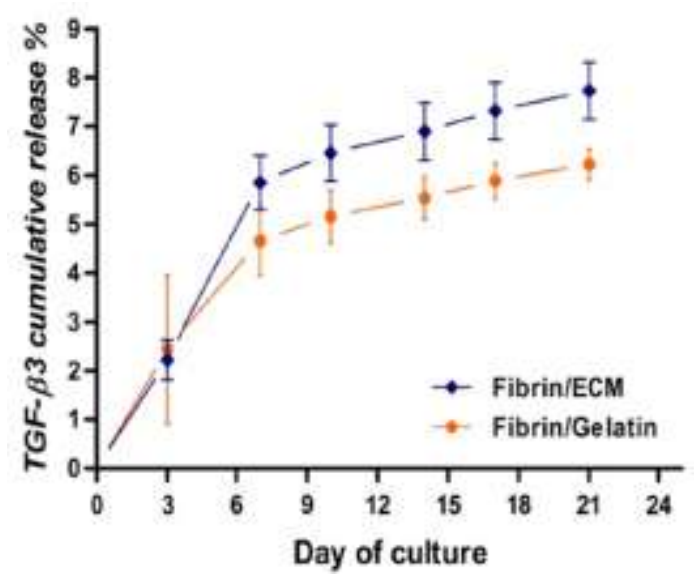

Fig 4

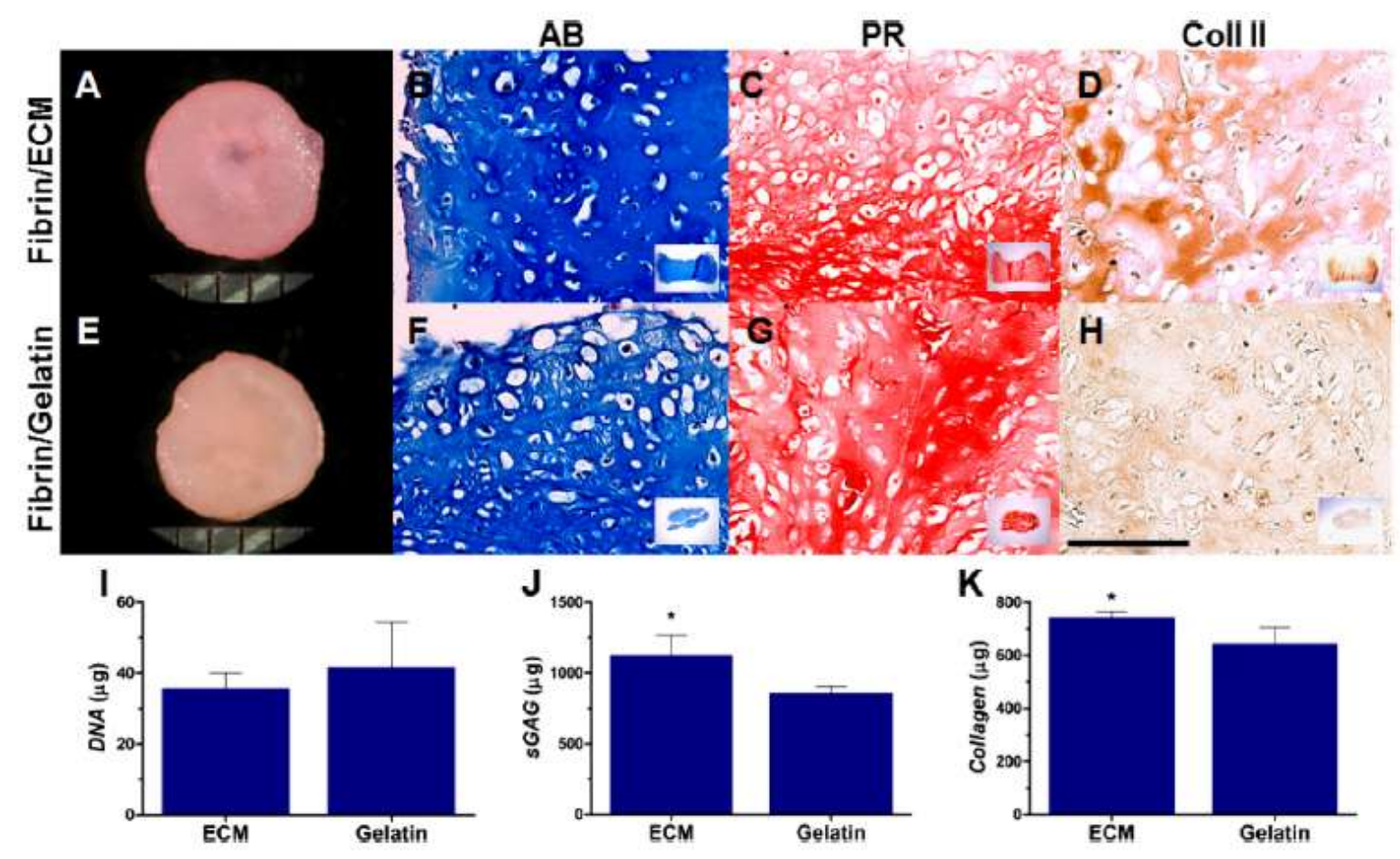


Fig 5

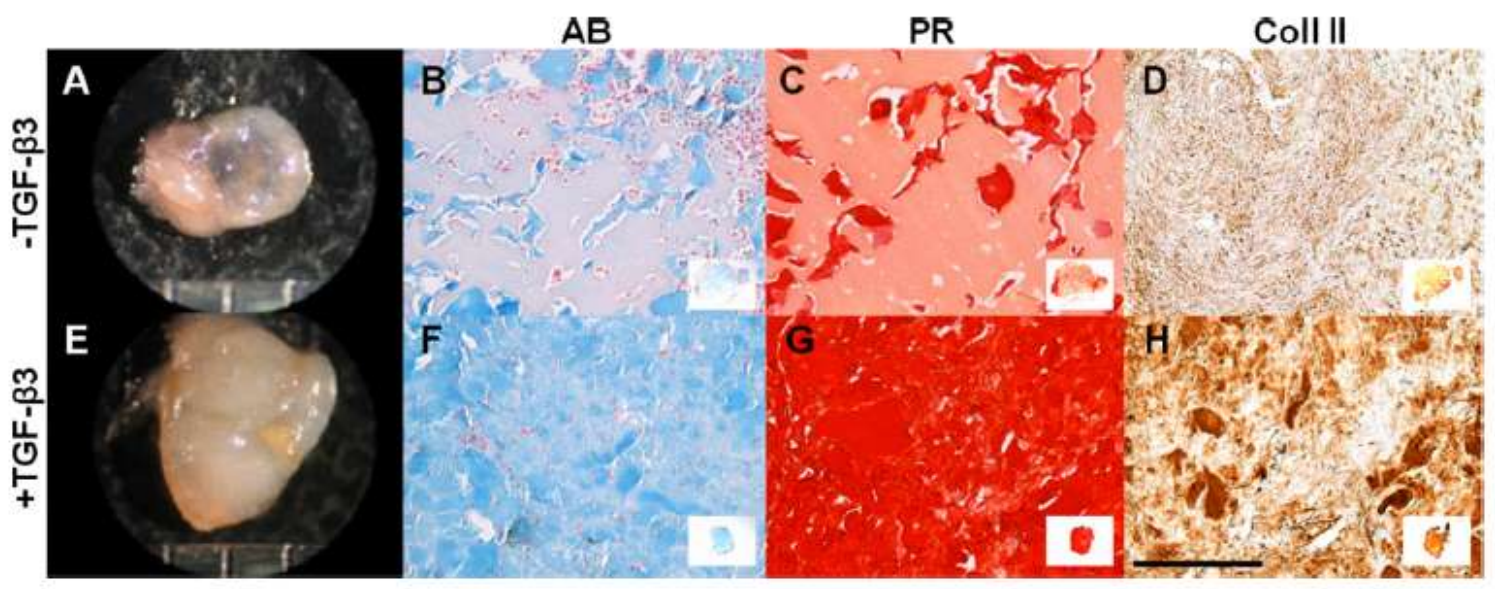

Fig 6
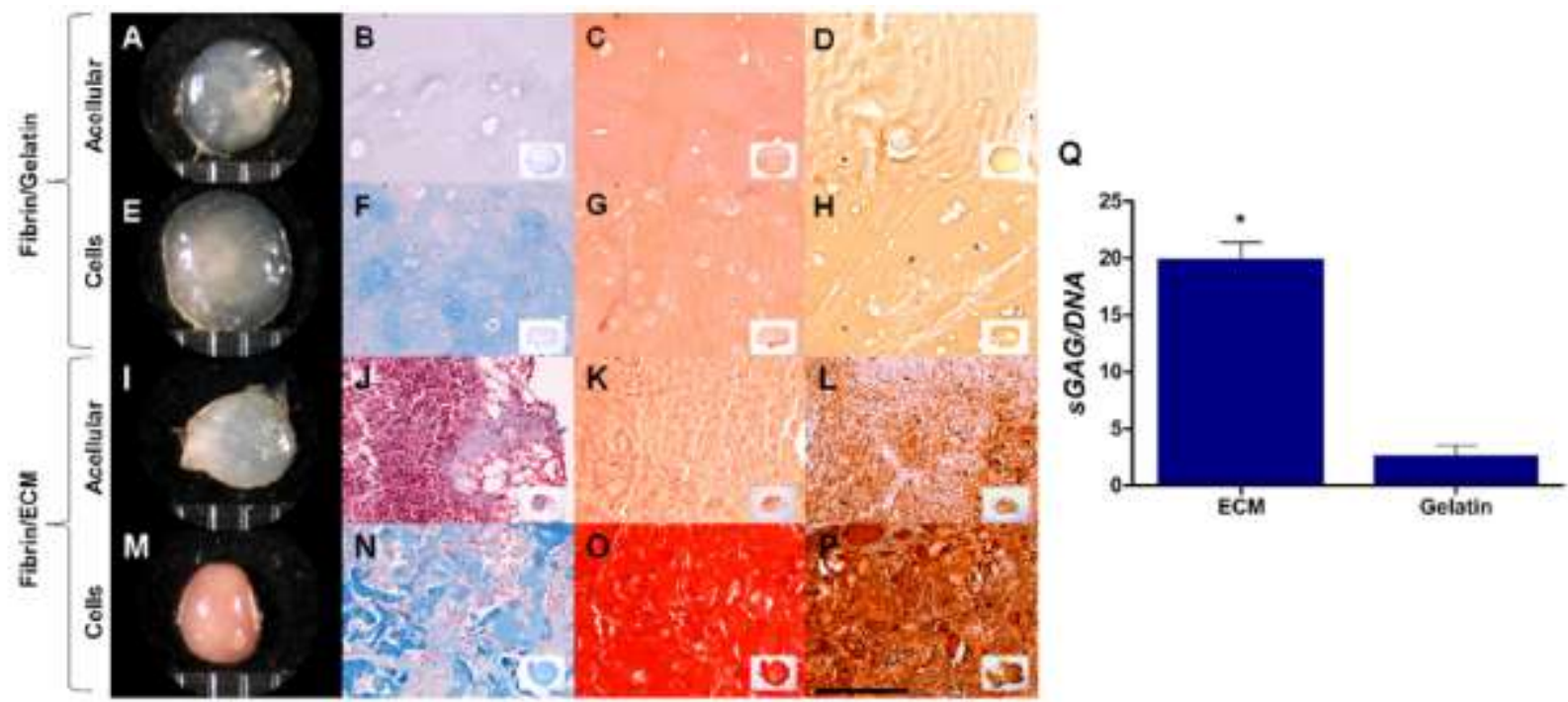